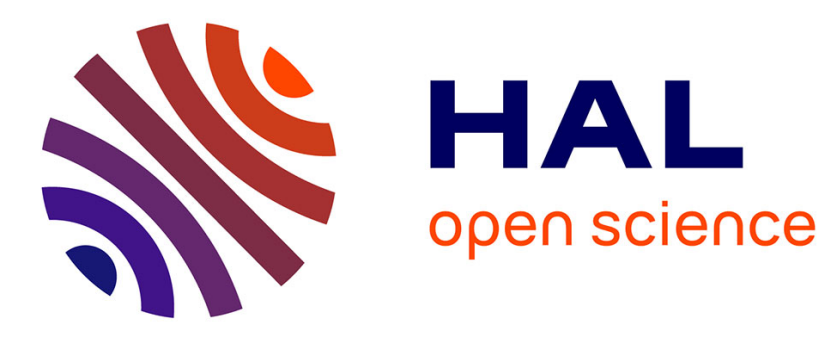

\title{
Photoinduction of surface-relief gratings during all-optical poling of polymer films
}

Aleksandra Apostoluk, Jean-Michel Nunzi, Céline Fiorini-Debuisschert

\section{To cite this version:}

Aleksandra Apostoluk, Jean-Michel Nunzi, Céline Fiorini-Debuisschert. Photoinduction of surfacerelief gratings during all-optical poling of polymer films. Optics Letters, 2004, 29 (1), pp.98. 10.1364/OL.29.000098 . hal-01988462

\section{HAL Id: hal-01988462 https://hal.science/hal-01988462}

Submitted on 17 Jan 2022

HAL is a multi-disciplinary open access archive for the deposit and dissemination of scientific research documents, whether they are published or not. The documents may come from teaching and research institutions in France or abroad, or from public or private research centers.
L'archive ouverte pluridisciplinaire HAL, est destinée au dépôt et à la diffusion de documents scientifiques de niveau recherche, publiés ou non, émanant des établissements d'enseignement et de recherche français ou étrangers, des laboratoires publics ou privés.

\section{(ㄷ)(1) $\$$}

Distributed under a Creative Commons Attribution - NonCommerciall 4.0 International 


\title{
Photoinduction of surface-relief gratings during all-optical poling of polymer films
}

\author{
Aleksandra Apostoluk and Jean-Michel Nunzi \\ Laboratoire des Propriétés Optiques des Matériaux et Applications, Unité Mixte de Recherche-Centre National de la Recherche \\ Scientifique 6136, Université d'Angers, 2 Boulevard Lavoisier, 49045 Angers, France \\ Céline Fiorini-Debuisschert \\ Commissariat à l'Energie Atomique, Direction de la Recherche Technologique, Laboratoire d'Intégration des Systèmes et \\ des Technologies, Département d'Elaboration et de Contrôle des Structures, Service d'Elaboration et \\ de Mise en Forme des Matériaux, Laboratoire Composants Organiques, Centre d'Etudes de Saclay, 91191 Gif-sur-Yvette, France
}

\begin{abstract}
Using the all-optical poling method, we observed the formation of a surface-relief grating (SRG) in an amorphous polymer film containing azo dye molecules in side chain positions. The experiment consists of a seeding-type process. We used a recently described experimental setup that permits a periodic nonlinear pattern to be produced by the index dispersion of glass. The particular configuration permits photoinduced translation diffusion of the azo chromophores to be observed as the origin of the SRG formation. Analyses of the gratings recorded by use of $s$ (TE) and $p$ (TM) polarization of the writing beams are conducted by atomic-force microscopy. The effect is attributed to mass transport from regions of high isomerization activity to regions of lower activity.
\end{abstract}

Since the discovery of self-organized second-harmonic generation (SHG) in optical fibers, ${ }^{1,2}$ much effort has been dedicated to the achievement of noncentrosymmetric orders in various materials by purely optical means., ${ }^{3,4}$ In the all-optical poling method, noncentrosymmetric molecular orientation appears in polymers owing to the presence of second-order nonlinear susceptibility $\chi^{(2)}$ gratings formed by the nonzero temporal average cube of the coherent interference between fundamental and second-harmonic (SH) beams. ${ }^{5}$ In terms of photophysics, the molecules are excited by both fundamental and $\mathrm{SH}$ fields. They undergo trans-cis isomerization, followed by reorientation and relaxation to the trans ground state, until they reach an equilibrium between polar excitation and orientation relaxation, leaving a net $\chi^{(2)}$ susceptibility inside the material. ${ }^{6}$ In theory as well as experimentally, ${ }^{5}$ the induced birefringence should not be periodically modulated. However, Tsutsumi et al. ${ }^{7}$ observed diffraction gratings created in the medium by means of all-optical poling. They assigned it to a birefringence that oscillates with a double period compared with the $\chi^{(2)}$ grating. The formation of a surface-relief grating (SRG) in these conditions is not trivial, as neither a polarization nor an intensity grating is present. ${ }^{8,9}$ Formation of the SRG with subsequent corona poling to produce second-order nonlinearity has already been reported. ${ }^{10}$

We investigated the physical properties of the second-order susceptibility $\chi^{(2)}$ gratings induced in amorphous azo polymer films. We found that SRGs can be locally induced simultaneously with the $\chi^{(2)}$ gratings. To our knowledge this is the first demonstration of the concurrent formation of $\chi^{(2)}$ and SRGs.

Samples used in this study were spin-coated thin films of the azo molecules Disperse Red 1 grafted onto a poly(methyl methacrylate) skeleton with $35 \%$ molar concentration. Samples were $\sim 200 \mathrm{~nm}$ thick, with an optical density of 0.8 at $532 \mathrm{~nm}$. The experimental setup was described previously ${ }^{11}$ : The laser source is a Quantel $Q$-switched Nd:YAG laser that delivers 7-ns pulses at $1064 \mathrm{~nm}$ with a $10-\mathrm{Hz}$ repetition rate. The fundamental beam is partially doubled in frequency by an external-cavity KDP crystal. Fundamental and SH writing beam fluences are optimized to reach maximum poling efficiency. A polarizer ensures parallel polarization of the two beams. The polarization direction ( $p$ and $s$ polarization) of the writing beams is controlled with a half-wave plate. Both polarization configurations are used in our experiments. The prism on which a sample is placed permits one to prevent the relative phase differences between the writing beams at frequencies $\omega$ and $2 \omega$ to influence the efficiency of the all-optical poling. ${ }^{11} p$ and $s$ polarization refers to the plane perpendicular to the edge of the prism: TM and TE, respectively. Writing and probing periods alternate. During writing periods, the two writing beams at frequencies $\omega$ and $2 \omega$ simultaneously impinge onto the sample. For probing, the writing period is interrupted at regular intervals by insertion of a green-blocking RG-630 Schott filter, leaving only the $\omega$ beam as a probe of SHG. The signal is collected by a photomultiplier tube and averaged by a digital oscilloscope. A shutter in front of the photomultiplier tube is open in synchronization with the insertion of the green-blocking filter. It is used to protect the photomultiplier tube from damage caused by the high-energy seeding beam at $2 \omega$ frequency. A set of calibrated filters is also used to ensure correct scaling of the SH signal. The average beam intensities were 2 and $1.7 \mathrm{~mW} / \mathrm{cm}^{2}$ for the fundamental and the $\mathrm{SH}$ beams, respectively. 
Photoinduced second-order susceptibility $\chi^{(2)}$ can be probed by use of SHG from the sample. The typical growth of the SH signal recorded for $s$ and $p$ seeding beam polarization configurations is presented in Fig. 1. It can be seen that the values of the $\mathrm{SH}$ intensity obtained in the two cases are identical. The induced $\chi^{(2)}$ gratings also decay in the same way. This proves that the use of the prism in the experimental setup does not influence the nature of the seeding process, ${ }^{5}$ so the longitudinal $\chi^{(2)}$ grating induced in the thin film ${ }^{11}$ is not affected by the $s$ or $p$ nature of the linear seeding polarization.

The samples were illuminated for several hours $(\approx 1$ day), as the green seeding beam's intensity was relatively low. The idea was to irradiate the sample with almost the same amount of light $\left(>100 \mathrm{~J} / \mathrm{cm}^{2}\right)$ as is typically absorbed during the encoding of SRGs. ${ }^{12-14}$ The gratings were recorded with both $s$ and $p$ seeding beam polarizations. The surface profiles were measured by atomic-force microscopy (AFM) in the contact mode. We observed SRG formation with a period of $\sim 9 \mu \mathrm{m}$ along the $\chi^{(2)}$ grating's direction (perpendicular to the prism's edge). This period is half the period of the second-order susceptibility $\chi^{(2)}$ gratings encoded into the medium. ${ }^{11}$ The AFM scans and surface profiles for $p$ polarization (28-h irradiation) and $s$ polarization (46-h irradiation) are presented in Figs. 2(a) and 2(b), respectively. The peak-to-valley modulation amplitude was $\Delta h \approx 25 \mathrm{~nm}$ for the $p$ polarization and $\Delta h \approx 12 \mathrm{~nm}$ for the $s$ polarization; a factor of 3 difference between them could be expected theoretically. ${ }^{12}$ These modulation values are significant, as we use a weak green beam intensity of $1.7 \mathrm{~mW} / \mathrm{cm}^{2}$. The presence of thermal effects in the mass transport process can be excluded owing to the low intensity of light and to the dependence of the surface modulation amplitude induced on the writing beams' polarization. Additionally, the laser energy level is much below the ablation threshold of the polymer. In our experiments, the light's intensity as well as its polarization is not modulated along the grating. Therefore any field gradient is excluded as the origin of the mass transport observed. ${ }^{15}$ It appears experimentally that $p$ polarization is more efficient than $s$ polarization in inducing surface relief. This was predicted for the nonthermal photoinduced molecular migration model based on the anisotropy of the photoinduced translation diffusion of azo benzene dyes. ${ }^{12}$ The surface relief turned out to be quasipermanent, as was verified by AFM a long time after the grating was written. However, we did not explicitly probe the erasure behavior of the gratings. To investigate the effect of the incident light power, we performed the experiment with increased seeding beam intensities: as much as $I_{\omega}=3$ and $I_{2 \omega}=4 \mathrm{~mW} / \mathrm{cm}^{2}$, close to the ablation limit of the polymer. After $16 \mathrm{~h}$ of illumination under $p$ polarization, the surface modulation reached $\Delta h \approx 35 \mathrm{~nm}$.

We propose the following physical explanation for the phenomenon of SRG formation during dualfrequency interference (see Fig. 3). The molecular orientation distribution is initially isotropic and centrosymmetric. The photoexcited molecules rotate under the action of light. They tend to reduce their interaction with the incident field such that they align antiparallel to the direction of the cube of the incident field $\left\langle E^{3}\right\rangle_{t} \cdot{ }^{4}$ After a typical period of $1 \mathrm{~h}$ (Fig. 1) the molecular distribution of orientations becomes noncentrosymmetric. This means that the number of molecules oriented parallel and antiparallel to the field is unequal and that only this contributes to the encoding of second-order nonlinear susceptibility in the medium. Owing to our arrangement that uses a dispersion prism, ${ }^{11}$ the polar term of the probability of excitation that is responsible for the formation of the $\chi^{(2)}$ grating alternates between positive and

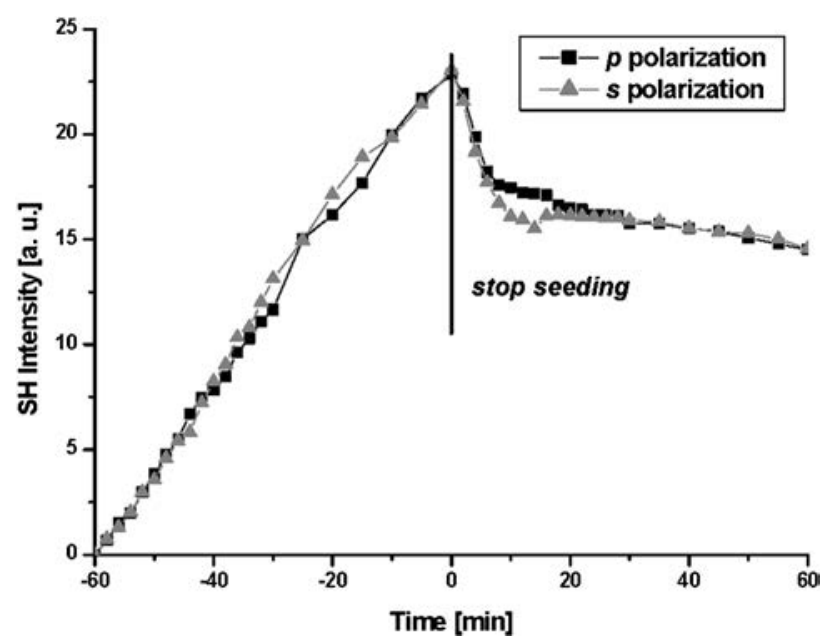

Fig. 1. Real-time growth and lifetime of the SH generated signal (in arbitrary units) in $p$-polarization and $s$-polarization writing beam configurations. Negative times correspond to the seeding preparation phase. At time zero the seeding is stopped. Positive times are related to the study of the temporal decay of the photoinduced $\chi^{(2)}$ susceptibility.
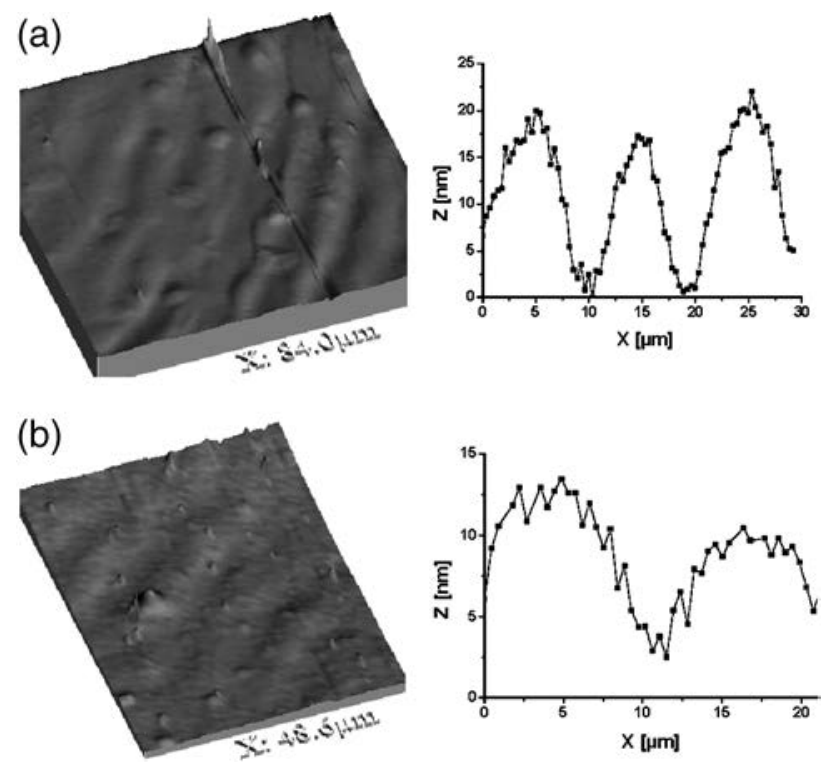

Fig. 2. AFM topographic image and cross section of a SRG induced (a) after $28 \mathrm{~h}$ of irradiation in a Disperse Red $1 /$ poly(methyl methacrylate) $35 / 65$ thin film in a $p$-polarization writing beam configuration and (b) after $46 \mathrm{~h}$ of irradiation in an $s$-polarization configuration. 


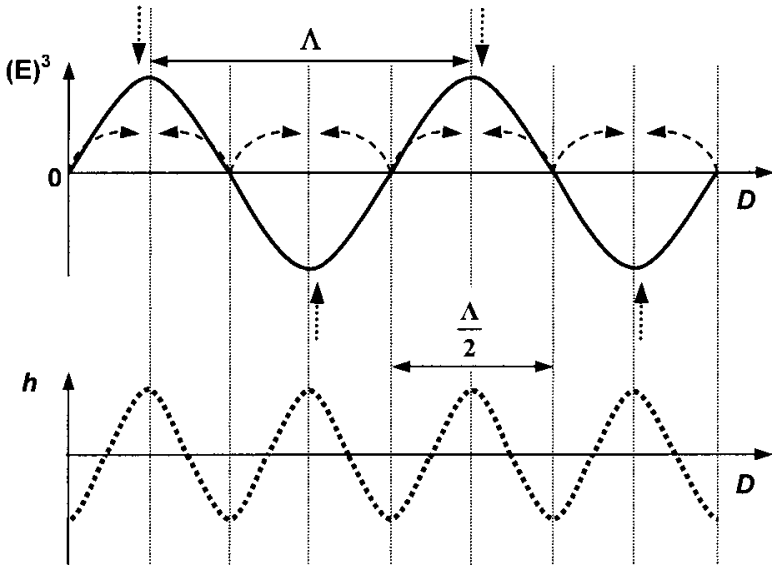

Fig. 3. Schematic of the creation of a SRG by all-optical poling. Continuous curve, modulation of the temporal average cube $\left\langle E^{3}\right\rangle_{t}$ of the polar field with period $\Lambda$ (the polar field is a coherent superposition of two beams at fundamental and SH frequencies). Dotted arrows, antiparallel orientation (under $s$ polarization) of molecules to minimize their interaction with the incident field. Dashed arrows, directions of molecular migration once the molecular orientation distribution reaches its stationary level. Dotted curve, modulation $h$ of the sample surface with period $\Lambda / 2$, induced by photoinduced mass transport. $D$, diameter of the interaction region.

negative maxima with a period $\Lambda$ in the plane of the sample placed on top of the prism. The SHG grating wave vector is $\mathbf{K}_{\mathrm{SHG}}=2 \mathbf{k}_{\omega}-\mathbf{k}_{2 \omega}$, where $\mathbf{k}_{\omega}$ and $\mathbf{k}_{2 \omega}$ refer to the fundamental and harmonic wave vectors, respectively. Hence a periodic quasi-phase-matched structure with domain inversion is created in the polymer film. Once the process of polar orientation is completed, oscillation of $\chi^{(2)}$ means that there are places where $\chi^{(2)}$ is zero. These are unpoled regions: nodes that are present with a doubled spatial frequency $\Lambda / 2$. These nodes are regions with an increased amount of isomerization activity. There, the molecules are excited with much higher efficiency than in the areas in which the polar term attains its maxima (negative or positive) once the induced polar orientation is photostationary and the second-order susceptibility $\chi^{(2)}$ grating is encoded. This improved excitation causes mass transport from regions of high to lower isomerization activity. After several hours of irradiation, as pictured in Fig. 3, the SRG is inscribed with a half-period $(\sim 9 \mu \mathrm{m})$ compared with the periodicity of the $\chi^{(2)}$ grating $(17 \pm 1 \mu \mathrm{m}$ was calculated from the refractive indices of the prism ${ }^{11}$ ). The SRG wave vector is $\mathbf{K}_{\mathrm{SRG}}=4 \mathbf{k}_{\omega}-2 \mathbf{k}_{2 \omega}$. It is worth mentioning that, if a unidirectional migration of molecules is possible, ${ }^{16}$ it should not be observed under the conditions of all-optical poling with dual-frequency fields. Indeed, the orientation photostationary state is achieved in a period of time that is at least 1 order of magnitude smaller than the typical time to construct a surface relief, ${ }^{12}$ so the molecules that undergo the largest translation activity lie finally in nonpolar regions. The signature of a unidirectional migration should have been $\mathbf{K}_{\mathrm{SRG}}=\mathbf{K}_{\mathrm{SHG}}$, an issue that we intend to investigate in more detail in further studies by use of $\mathrm{SH}$ microscopy correlated locally with the surface relief. ${ }^{17-20}$

We have shown that both second-order nonlinear susceptibility $\chi^{(2)}$ gratings and surface-relief gratings can be inscribed in azo polymer thin films by purely optical means. The mechanism that is responsible for surface-relief formation is photoisomerization-driven mass transport in a molecular thin film with periodic polar orientation. This is a new specific feature of dual-frequency interference. However, no unidirectional motion can be selected under such conditions.

We thank P. Raimond [Commissariat á l'Energie Atomique (CEA) Saclay] for synthesis of the copolymer, P. Saulnier (Faculté de Pharmacie, Université d'Angers), and C. Hubert (CEA Saclay) for the AFM scans. J.-M. Nunzi's e-mail address is jeanmichel.nunzi@univ-angers.fr.

\section{References}

1. U. Österberg and W. Margulis, Opt. Lett. 11, 516 (1986).

2. R. H. Stolen and H. W. K. Tom, Opt. Lett. 12, 585 (1987).

3. T. J. Driscoll and N. M. Lawandy, J. Opt. Soc. Am. B 11, 355 (1994).

4. F. Charra, F. Kajzar, J.-M. Nunzi, P. Raimond, and E. Idiart, Opt. Lett. 18, 941 (1993).

5. C. Fiorini, F. Charra, J.-M. Nunzi, and P. Raimond, J. Opt. Soc. Am. B 14, 1984 (1997).

6. C. Fiorini and J. M. Nunzi, Chem. Phys. Lett. 286, 415 (1998).

7. N. Tsutsumi, J. Yamamoto, and W. Sakai, Jpn. J. Appl. Phys. 40, 2264 (2001).

8. P. Rochon, E. Batalla, and A. Natansohn, Appl. Phys. Lett. 66, 136 (1995).

9. D. Y. Kim, S. K. Tripathy, L. Li, and J. Kumar, Appl. Phys. Lett. 66, 1166 (1995).

10. Y. Che, O. Sugihara, C. Egami, H. Fujimura, Y. Kawata, N. Okamoto, M. Tsuchimori, and $\mathrm{O}$. Watanabe, Jpn. J. Appl. Phys. 38, 6316 (1999).

11. A. Apostoluk, D. Chapron, C. Fiorini-Debuisschert, G. Gadret, J.-M. Nunzi, P. Raimond, and B. Sahraoui, Opt. Lett. 27, 2028 (2002).

12. P. Lefin, C. Fiorini, and J.-M. Nunzi, Pure Appl. Opt. 7, 71 (1998).

13. I. Naydenova, L. Nikolova, T. Todorov, N. R. C. Holme, P. S. Ramanujam, and S. Hvilsted, J. Opt. Soc. Am. B 15, 1257 (1998).

14. X. L. Jiang, L. Li, J. Kumar, D. Y. Kim, and S. K. Tripathy, Appl. Phys. Lett. 27, 2502 (1998).

15. J. Kumar, L. Li, X. L. Jiang, D. Y. Kim, T. S. Lee, and S. K. Tripathy, Appl. Phys. Lett. 72, 2096 (1998).

16. N. Sertova, I. Petkov, C. Fiorini, P. Raimond, and J.-M. Nunzi, Mol. Cryst. Liq. Cryst. 374, 77 (2002).

17. M. Adameck, R. Blum, and M. Eich, Appl. Phys. Lett. 73, 2884 (1998).

18. G. Martin, E. Toussaere, L. Soulier, and J. Zyss, Synth. Metals 127, 49 (2002).

19. S. Kluge, F. Budde, I. Dohnke, P. Rechsteiner, and J. Hulliger, Appl. Phys. Lett. 81, 247 (2002).

20. M. Kobayashi, K. Fujita, T. Kaneko, T. Takamatsu, O. Nakamura, and S. Kawata, Opt. Lett. 27, 1324 (2002). 